\title{
The consensus value: a new solution concept for cooperative games
}

\author{
Yuan Ju • Peter Borm • Pieter Ruys
}

Received: 20 February 2006 / Accepted: 9 August 2006 /

Published online: 13 September 2006

(C) Springer-Verlag 2006

\begin{abstract}
To generalize the standard solution for 2-person TU games into $n$-person cases, this paper introduces a recursive two-sided negotiation process to establish cooperation between all players. This leads to a new solution concept for cooperative games: the consensus value. An explicit comparison with the Shapley value is provided, also at the axiomatic level. Moreover, a class of possible generalizations of the consensus value is introduced and axiomatized with the Shapley value at one end and the equal surplus solution at the other. Finally, we discuss a non-cooperative mechanism which implements the consensus value.
\end{abstract}

\section{Introduction}

In this paper, we investigate a new solution concept for sharing the joint gains of cooperation based on an explicit recursive two-sided negotiation process. In this respect we conceptually generalize the standard solution for 2-person games

\footnotetext{
$\mathrm{Y} . \mathrm{Ju}(\varangle)$

School of Economic and Management Studies, Keele University, Keele, Staffordshire ST5 5BG, UK

e-mail: Y.Ju@keele.ac.uk

P. Borm

CentER and Department of Econometrics and Operations Research,

Tilburg University, Tilburg, The Netherlands

P. Ruys

TILEC, Department of Econometrics and Operations Research, and Tias Business School, Tilburg University, Tilburg, The Netherlands
} 
into $n$-person cases and obtain a new solution concept for TU (transferable utility) games: the consensus value.

The standard solution for 2-person games assigns to each player her individual coalitional value and distributes the remainder, i.e., the (net) surplus, of the value of the grand coalition equally between the two players. Following this "standard" idea, we introduce a two-sided negotiation process to establish cooperation in $n$-person games. Given an ordering of the players, we take a bilateral perspective and consider that any surplus is the joint contribution between an existing coalition of players (i.e., the incumbents) and an entrant. By taking the incumbents as one party and the entrant as a second party, the standard solution for 2-person games can be applied all the way with consensus. That is, all the joint surpluses are always equally split between the corresponding two parties. Since no specific ordering is pre-determined, we average over all possible permutations.

Such a constructive process is helpful to solve practical problems. Ju et al. (2004) argue in favor of such a process to study loss compensation and surplus sharing problems in so-called project-allocation situations. A similar process is defined in the context of bankruptcy problems in order to extend the generally accepted concede-and-divide rule (cf. Quant et al. 2005). The consensus idea has also been applied in resolving conflicts and in reaching compromise outcomes in economic environments with externalities. In this setting the solution concept well balances the coalitional effects and externality effects (cf. Ju 2004). Furthermore, the definition of the concession rule, a rule to solve the compensation problem associated with the issue of (inter-individual) externality, is also inspired by the consensus idea (cf. Ju and Borm 2005).

The Shapley value (Shapley 1953) can be introduced via a similar procedural definition, i.e., by the marginal contribution approach. In our opinion, however, the marginal contribution approach, from the point of view of negotiations, is not so convincing. More specifically, the terminology of "marginal contribution" is somewhat misleading. In fact, the marginal contribution is jointly created by the existing coalition of players and the entrant, not by the entrant solely. Of course, in the Shapley value itself, this aspect is smoothed out by taking the average of the marginal contributions over all different orders.

As an illustration for the consensus value, we consider a particular class of glove games where the consensus value performs well. Moreover, we will explicitly describe the relations between the consensus value and the Shapley value, also on the axiomatic level. As can be expected the main difference is in the treatment of dummy players.

We characterize the consensus value as the unique one-point solution concept for TU games that satisfies efficiency, symmetry, the neutral dummy property and additivity. The neutral dummy property balances the tensions between the four fundamental principles of distributive justice (cf. Moulin 2003). One is the reward principle, distributing resources within a society based on the members' contributions, which, therefore, implies that dummy players cannot obtain more than their stand-alone payoffs. A second is the fitness principle, aiming at maximizing the sum of the members' utilities (by giving resources to the most 
capable ones) and requiring a dummy to get the stand-alone payoff only. These two principles support the classical dummy property. A third is the compensation principle, aiming at maximizing the minimum utility of the members of a society, and therefore, justifies unequal shares of resources in order to achieve equality ex post. A fourth is the exogenous rights principle, corresponding to equality ex ante, in the sense that all members of a society have an equal claim to its resources. The last two principles support equal sharing of total revenues also for dummy players. The neutral dummy property offers a comparison between these principles.

By means of the transfer property, a second characterization for the consensus value is provided. Using an equal welfare loss property which is similar to the balanced contribution property a la Myerson (1980), a third characterization is obtained. We also establish two recursive formulas for the consensus value: One is based on a stand-alone reduced game while the other uses the classical notion of subgames. Moreover and rather surprisingly, we find that the consensus value is the average of the Shapley value and the equal surplus solution (cf. Moulin 2003) which is also known as the CIS-value (Driessen and Funaki 1991; van den Brink and Funaki 2004). Based on this result, we turn back to examine the specific links between sharing procedures and final outcomes, which implies that we can generalize the consensus value in a natural way. In particular, the Shapley value and the equal surplus solution turn out to be the two polar cases of these generalized consensus values. We then provide characterizations for the class of generalized consensus values. Finally, the consensus value is studied from a non-cooperative perspective: We introduce a variant of the bidding mechanism proposed by Pérez-Castrillo and Wettstein (2001) and show that it implements the consensus value in subgame perfect equilibrium.

In the next section, we provide the formal definition of the consensus value together with two recursive formulas. Section 3 focuses on the relation with the Shapley value, primarily at the axiomatic level. A generalization of the consensus value is provided in this section too. The final section discusses an implementation of the consensus value.

\section{The consensus value}

Let us consider an arbitrary 2-person cooperative TU game with player set $N=\{1,2\}$ and characteristic function $v$ determined by the values: $v(\{1\}), v(\{2\})$ and $v(\{1,2\})$. A reasonable solution is that player 1 gets

$$
v(\{1\})+\frac{v(\{1,2\})-v(\{1\})-v(\{2\})}{2}
$$

and player 2 gets

$$
v(\{2\})+\frac{v(\{1,2\})-v(\{2\})-v(\{1\})}{2} .
$$


That is, the (net) surplus generated by the cooperation between player 1 and $2, v(\{1,2\})-v(\{1\})-v(\{2\})$, is equally shared between the two players. This solution is called the standard solution for 2-person cooperative games.

Now, we provide a generalization of the standard solution for 2-person games into $n$-person cases. It follows the following line of reasoning.

Consider a 3-person game $(N, v)$ with player set $N=\{1,2,3\}$. Assume we have the order $(1,2,3)$ : player 1 shows up first, then player 2 , and finally player 3 . When player 2 joins player 1, we in fact have a 2-person situation, and following the principles of the standard solution, the surplus $v(\{1,2\})-v(\{1\})-v(\{2\})$ will be equally split between them.

Next player 3 enters the scene, who would like to cooperate with player 1 and 2. Because coalition $\{1,2\}$ has already been formed before she enters the game, player 3 will actually cooperate with the existing coalition $\{1,2\}$ instead of simply cooperating with 1 and 2 individually. If $\{1,2\}$ agrees to cooperate with 3 as well, the coalitional value $v(\{1,2,3\})$ will be generated. Now, the question is how to share it?

Again, following the standard solution to 2-person games, one can argue that both parties should get half of the joint surplus $v(\{1,2,3\})-v(\{1,2\})-v(\{3\})$ in addition to their individual coalitional values. The reason is simple: coalition $\{1,2\}$ can be regarded as one player instead of two players because they have already formed a cooperating coalition. Internally, 1 and 2 will receive equal shares of the surplus because this part is obtained extra by the coalition $\{1,2\}$ cooperating with coalition $\{3\}$.

One can also tell this story in the reverse way, yielding the same outcome in terms of surplus sharing. Initially, three players cooperate with each other and $v(\{1,2,3\})$ is obtained. We now consider players leaving the existing coalition one by one in the opposite order $(3,2,1)$. So, player 3 leaves first. As a consequence, the grand coalition partitions into two parts: $\{1,2\}$ and $\{3\}$. How much should player 3 take from the value $v(\{1,2,3\})$ as she leaves? No doubt these two parties should first get $v(\{1,2\})$ and $v(\{3\})$, respectively. As for the surplus/loss $v(\{1,2,3\})-v(\{3\})-v(\{1,2\})$, one can argue that since player 3 is now negotiating with the coalition $\{1,2\}$ as a whole and the surplus/loss is jointly created by the two parties, the standard solution for 2-person games can be applied and each party should then get half of the joint surplus/loss. Consequently, player 3 obtains half of the joint surplus/loss plus her individual payoff, i.e.,

$$
v(\{3\})+\frac{v(\{1,2,3\})-v(\{3\})-v(\{1,2\})}{2},
$$

and the remaining goes to the cooperating coalition $\{1,2\}$. Thus, the value left for coalition $\{1,2\}$, which we call the standardized remainder (the value left for the corresponding remaining coalition) for $\{1,2\}$, is

$$
v(\{1,2\})+\frac{v(\{1,2,3\})-v(\{1,2\})-v(\{3\})}{2} .
$$


In the same fashion, the standardized remainder for $\{1\}$ will be

$$
v(\{1\})+\frac{\left(v(\{1,2\})+\frac{v(\{1,2,3\})-v(\{1,2\})-v(\{3\})}{2}\right)-v(\{1\})-v(\{2\})}{2}
$$

when player 2 leaves the coalition $\{1,2\}$ next, while player 2 will obtain

$$
v(\{2\})+\frac{\left(v(\{1,2\})+\frac{v(\{1,2,3\})-v(\{1,2\})-v(\{3\})}{2}\right)-v(\{2\})-v(\{1\})}{2} .
$$

Extending this argument to an $n$-person case, we obtain a general method, which can be understood as a standardized remainder rule since we take the later entrant (or earlier leaver) and all her pre-entrants (or post-leavers) as two parties and apply the standard solution for 2-person games all the way. Furthermore, since no ordering is pre-determined for a TU game, we will average over all possible orderings.

Formal definitions are provided below. ${ }^{1}$ We denote by $T U^{N}$ the class of all TU games with player set $N$. Let $\Pi(N)$ be the set of all bijections $\sigma$ : $\{1, \ldots,|N|\} \longrightarrow N$. For a given $\sigma \in \Pi(N)$ and $k \in\{1, \ldots,|N|\}$ we define $S_{k}^{\sigma}=\{\sigma(1), \ldots, \sigma(k)\} \subset N$ and $S_{0}^{\sigma}=\emptyset$. Let $v \in T U^{N}$. Recursively, we define

$r\left(S_{k}^{\sigma}\right)= \begin{cases}v(N) & \text { if } k=|N| \\ v\left(S_{k}^{\sigma}\right)+\frac{1}{2}\left(r\left(S_{k+1}^{\sigma}\right)-v\left(S_{k}^{\sigma}\right)-v(\{\sigma(k+1)\})\right) & \text { if } k \in\{1, \ldots,|N|-1\}\end{cases}$

where $r\left(S_{k}^{\sigma}\right)$ is the standardized remainder for coalition $S_{k}^{\sigma}$ : the value left for $S_{k}^{\sigma}$ after allocating surpluses to earlier leavers $N \backslash S_{k}^{\sigma}$.

We then construct the individual standardized remainder vector $s^{\sigma}(v)$, which corresponds to the situation where the players leave the game one by one in the order $(\sigma(|N|), \ldots, \sigma(1))$ and assign to each player $\sigma(k)$, besides her individual payoff $v(\{\sigma(k)\})$, half of the net surplus from the standardized remainder $r\left(S_{k}^{\sigma}\right)$. Formally,

$$
s_{\sigma(k)}^{\sigma}(v)= \begin{cases}v(\{\sigma(k)\})+\frac{1}{2}\left(r\left(S_{k}^{\sigma}\right)-v\left(S_{k-1}^{\sigma}\right)-v(\{\sigma(k)\})\right) & \text { if } k \in\{2, \ldots,|N|\} \\ r\left(S_{1}^{\sigma}\right) & \text { if } k=1\end{cases}
$$

Definition 1 For every $v \in T U^{N}$, the consensus value $\Psi(v)$ is defined as the average, $^{2}$ over the set $\Pi(N)$ of all bijections, of the individual standardized

\footnotetext{
1 We want to note that a definition based on the forward way explained above can be constructed and its outcome is indeed equivalent to that of the reverse-way definition. However, for mathematical convenience and notational simplicity, we only provide the formal definition based on the reverse-way argument.

2 In fact, this means that the consensus value can be motivated from a non-cooperative bargaining perspective. That is, the underlying negotiation process can be formulated into an $|N|-1$ stage 2-party alternating-offer bargaining model a la Rubinstein (1982).
} 
remainder vectors, i.e.,

$$
\Psi(v)=\frac{1}{|N| !} \sum_{\sigma \in \Pi(N)} s^{\sigma}(v) .
$$

Hence, the consensus value can be interpreted as the expected individual standardized remainder a player can get by participating in coalitions.

Following the process of obtaining the consensus value, a more descriptive name for this solution concept could be the average serial standardized remainder value. ${ }^{3}$

The computational aspects of the consensus value are illustrated in the following example.

Example 1 Consider the 3-person TU game described below.

\begin{tabular}{c|ccccccc}
\hline$S$ & $\{1\}$ & $\{2\}$ & $\{3\}$ & $\{12\}$ & $\{13\}$ & $\{23\}$ & $\{123\}$ \\
$v(S)$ & 10 & 0 & 0 & 18 & 23 & 0 & 30 \\
\hline
\end{tabular}

With $\sigma:\{1,2,3\} \longrightarrow N$ defined by $\sigma(1)=2, \sigma(2)=1$ and $\sigma(3)=3$, which is shortly denoted by $\sigma=\left(\begin{array}{lll}2 & 1 & 3\end{array}\right)$, we get

$$
\begin{aligned}
& s_{3}^{\sigma}(v)\left(=s_{\sigma(3)}^{\sigma}(v)\right)=v(\{3\})+\frac{1}{2}(v(N)-v(\{1,2\})-v(\{3\}))=6, \\
& s_{1}^{\sigma}(v)\left(=s_{\sigma(2)}^{\sigma}(v)\right)=v(\{1\})+\frac{1}{2}(r(\{2,1\})-v(\{2\})-v(\{1\}))=17, \\
& s_{2}^{\sigma}(v)\left(=s_{\sigma(1)}^{\sigma}(v)\right)=r(\{2\})=v(\{2\})+\frac{1}{2}(r(\{2,1\})-v(\{2\})-v(\{1\}))=7 .
\end{aligned}
$$

All individual standardized remainder vectors are given by

\begin{tabular}{c|ccc}
\hline$\sigma$ & $s_{1}^{\sigma}$ & $s_{2}^{\sigma}$ & $s_{3}^{\sigma}$ \\
\hline$(123)$ & 17 & 7 & 6 \\
$(132)$ & $18 \frac{1}{4}$ & $3 \frac{1}{2}$ & $8 \frac{1}{4}$ \\
$(213)$ & 17 & 7 & 6 \\
$(231)$ & 20 & 5 & 5 \\
$(312)$ & $18 \frac{1}{4}$ & $3 \frac{1}{2}$ & $8 \frac{1}{4}$ \\
$(321)$ & 20 & 5 & 5 \\
\hline
\end{tabular}

Hence, $\Psi(v)=\left(18 \frac{5}{12}, 5 \frac{1}{6}, 6 \frac{5}{12}\right)$ whereas the Shapley value of this game is given by $\Phi(v)=\left(20 \frac{1}{6}, 3 \frac{2}{3}, 6 \frac{1}{6}\right)$.

\footnotetext{
3 In the same spirit, an alternative name for the Shapley value could be the average serial marginal contribution value. 
To further illustrate the consensus value, we consider a particular class of glove games.

Example 2 (A glove game) Let $N=\{1,2,3\}$ be the set of players. Player 1 has one left-hand glove. Player 2 and 3 have one right-hand glove each. A single glove is worth nothing. A (left-right) pair is worth 1 Euro. The corresponding TU game $(N, v)$ is determined by the values: $v(\{i\})=0$ for all $i \in N, v(\{2,3\})=0$, and $v(\{1,2\})=v(\{1,3\})=v(\{1,2,3\})=1$.

One can readily check that the consensus value of this game is $\Psi(v)=$ $\left(\frac{1}{2}, \frac{1}{4}, \frac{1}{4}\right)$, whereas the unique Core element and the Nucleolus of the game are $(1,0,0)$ and the Shapley value of this game is $\left(\frac{2}{3}, \frac{1}{6}, \frac{1}{6}\right)$.

In the more general case when $|N|>3$ with only one player having a lefthand glove, the consensus value yields that the left-hand glove player gets $\frac{1}{2}$ and each right-hand glove player gets $\frac{1}{2(|N|-1)}$. By contrast, the unique Core element or the Nucleolus assigns 1 to the left-hand glove player and the Shapley value $\frac{|N|-1}{|N|}$.

Please observe that the consensus value prescribes a rather natural and desired outcome. ${ }^{4}$ The realization of the value of 1 relies on one left-hand glove and one right-hand glove. Therefore, one could argue that each type should get $\frac{1}{2}$. By symmetry considerations, all the right-hand glove players will split the value of $\frac{1}{2}$ equally among themselves.

Note that one can construct additional examples on quota games or weighted majority games which have the similar flavor as the glove game.

The consensus value can be characterized by means of a recursive formula. Let $f: T U^{N} \longrightarrow \mathbb{R}^{N}$ be a solution concept. For $v \in T U^{N}$ and $i \in N$, we introduce the game $v^{-i} \in T U^{N \backslash\{i\}}$ defined by

$$
v^{-i}(S)= \begin{cases}v(S) & \text { if } S \varsubsetneqq N \backslash\{i\} \\ v(N \backslash\{i\})+\frac{v(N)-v(N \backslash\{i\})-v(\{i\})}{2} & \text { if } S=N \backslash\{i\},\end{cases}
$$

and call $v^{-i}$ the stand-alone reduced game of $v$ with respect to player $i$.

We say that a solution concept $f$ satisfies stand-alone recursion if for every game $v \in T U^{N}$ with $|N| \geq 3$ we have

$f_{i}(N, v)=\frac{1}{|N|}\left(\sum_{j \in N \backslash\{i\}} f_{i}\left(N \backslash\{j\}, v^{-j}\right)+\left(v(\{i\})+\frac{v(N)-v(N \backslash\{i\})-v(\{i\})}{2}\right)\right)$

for all $i \in N$.

4 We want to note that the consensus value depends on the answer to the question who is a player. In particular, anyone without any glove can obtain a positive payoff so long as she is considered to be a player. This potential drawback can be amended by assuming the set of players of a game is exogenously given. 
Proposition 1 The consensus value is the unique one-point solution concept on the class of all $n$-person TU games with $n \geq 2$ which is standard for 2-person games and satisfies stand-alone recursion.

Proof Clearly it suffices to prove that the consensus value satisfies stand-alone recursion. By Definition 1, one can check that

$$
\sum_{j \in N \backslash\{i\}} \Psi_{i}\left(N \backslash\{j\}, v^{-j}\right)=\frac{1}{(|N|-1) !} \sum_{\sigma \in \Pi(N) ; \sigma(|N|) \neq i} s_{i}^{\sigma}(v)
$$

and

$$
v(\{i\})+\frac{v(N)-v(N \backslash\{i\})-v(\{i\})}{2}=\frac{1}{(|N|-1) !} \sum_{\sigma \in \Pi(N) ; \sigma(|N|)=i} s_{i}^{\sigma}(v) .
$$

We now provide an alternative recursive formula by means of the usual subgames of $v$, denoted by $\left.v\right|_{S}$ (For each game $v$ and $S \subset N,\left.v\right|_{S}(T)=v(T)$ for any $T \subset S$ ), which is similar to the recursive formula for the Shapley value as described in Maschler and Owen (1989):

$$
\Phi_{i}(N, v)=\frac{1}{|N|} \sum_{j \in N \backslash\{i\}} \Phi_{i}\left(N \backslash\{j\},\left.v\right|_{N \backslash\{j\}}\right)+\frac{1}{|N|}(v(N)-v(N \backslash\{i\}))
$$

for all $v \in T U^{N}$ with $|N| \geq 2$ and $i \in N$. Without proof we state that

Proposition 2 The consensus value satisfies the following recursive formula. For all $v \in T U^{N}$ with $|N| \geq 2$,

$$
\begin{aligned}
\Psi_{i}(N, v)= & \frac{1}{|N|} \sum_{j \in N \backslash\{i\}}\left(\Psi_{i}\left(N \backslash\{j\},\left.v\right|_{N \backslash\{j\}}\right)+\frac{1}{|N|-1} \cdot \frac{v(N)-v(N \backslash\{j\})-v(\{j\})}{2}\right) \\
& +\frac{1}{|N|}\left(v(\{i\})+\frac{v(N)-v(N \backslash\{i\})-v(\{i\})}{2}\right)
\end{aligned}
$$

for all $i \in N$.

\section{Characterizations and generalizations}

\subsection{Characterizations}

Let $f: T U^{N} \longrightarrow \mathbb{R}^{N}$ be a one-point solution concept. We consider the following properties.

- Efficiency: $\sum_{i \in N} f_{i}(v)=v(N)$ for all $v \in T U^{N}$;

- Symmetry: for two players $i, j \in N$, if $v(S \cup\{i\})=v(S \cup\{j\})$ for any $S \subset N \backslash\{i, j\}$, then $f_{i}(v)=f_{j}(v)$ for all $v \in T U^{N}$; 
- The neutral dummy property: if for some player $i \in N, v(S \cup\{i\})=v(S)+$ $v(\{i\})$ for any $S \subset N \backslash\{i\}$, then $f_{i}(v)=\frac{1}{2} v(\{i\})+\frac{1}{2}\left(v(\{i\})+\frac{v(N)-\sum_{j \in N} v(\{j\})}{|N|}\right)$ for all $v \in T U^{N}$;

- Additivity: $f(v+w)=f(v)+f(w)$ for all $v, w \in T U^{N}$.

The properties of efficiency, symmetry, and additivity are clear by themselves. We will briefly discuss the neutral dummy property. Moulin (2003) discusses four fundamental principles of distributive justice: compensation, reward, exogenous rights, and fitness. Compensation and exogenous rights basically correspond to equality, which supports the view that all members of a society including dummies should share the joint surplus equally among them. On the contrary, reward and fitness justifies the classical utilitarianism, which requires no more value to be allocated to a dummy player $i$ than her own value $v(\{i\})$. Thus, deciding to assign a dummy player either $v(\{i\})$ or $v(\{i\})+\frac{v(N)-\sum_{j \in N} v((j\})}{|N|}$ provides a particular choice between contrastive principles of distributive justice. Balancing among these four extreme cases (in fact, boiling down to two polar opinions: utilitarianism and egalitarianism, or efficiency and equality), ${ }^{5}$ we make a fair compromise and take the average as the gain of a dummy player, which results in the so-called neutral dummy property.

We show that the consensus value is the unique one-point solution concept that satisfies the four properties discussed above. For this we first need the following lemma which states that the consensus value satisfies the basic property of relative invariance with respect to strategic equivalence.

Lemma 1 The consensus value $\Psi$ is relative invariant with respect to strategic equivalence, i.e., for $\alpha>0$ and $\beta \in \mathbb{R}^{N}$, we have

$$
\Psi_{i}(\alpha v+\beta)=\alpha \Psi_{i}(v)+\beta_{i}
$$

for all $i \in N$ and $v \in T U^{N}$.

Proof Let $v \in T U^{N}, \sigma \in \Pi(N), \alpha>0$ and $\beta \in \mathbb{R}^{N}$.

Claim $1 r^{\alpha v+\beta}\left(S_{k}^{\sigma}\right)=\alpha r^{v}\left(S_{k}^{\sigma}\right)+\sum_{i \in S_{k}^{\sigma}} \beta_{i}$ for all $k \in\{1,2, \ldots,|N|\}$.

For $k=|N|$, this is obvious.

5 Cultural and philosophical factors may affect the propensity or choice between the two polar opinions. 
For $k \in\{1, \ldots,|N|-1\}$, using induction we have

$$
\begin{aligned}
r^{\alpha \nu+\beta}\left(S_{k}^{\sigma}\right)= & \left(\alpha v\left(S_{k}^{\sigma}\right)+\sum_{i \in S_{k}^{\sigma}} \beta_{i}\right)+\frac{1}{2}\left(r^{\alpha v+\beta}\left(S_{k+1}^{\sigma}\right)\right. \\
& -\left(\alpha v\left(S_{k}^{\sigma}\right)+\sum_{i \in S_{k}^{\sigma}} \beta_{i}\right)-\left(\alpha v(\sigma(k+1))+\beta_{\sigma(k+1))}\right) \\
= & \alpha\left(v\left(S_{k}^{\sigma}\right)+\frac{1}{2}\left(r^{v}\left(S_{k+1}^{\sigma}\right)-v\left(S_{k}^{\sigma}\right)-v(\{\sigma(k+1)\})\right)\right)+\sum_{i \in S_{k}^{\sigma}} \beta_{i} \\
= & \alpha r^{v}\left(S_{k}^{\sigma}\right)+\sum_{i \in S_{k}^{\sigma}} \beta_{i}
\end{aligned}
$$

Claim $2 s_{\sigma(k)}^{\sigma}(\alpha v+\beta)=\alpha s_{\sigma(k)}^{\sigma}(v)+\beta_{\sigma(k)}$ for all $k \in\{1,2, \ldots,|N|\}$.

For $k \in\{2, \ldots,|N|\}$, by the definition of individual standardized remainder and Claim 1 we find

$$
\begin{aligned}
s_{\sigma(k)}^{\sigma}(\alpha v+\beta)= & \left.\alpha \nu(\sigma(k))+\beta_{\sigma(k)}\right) \\
& +\frac{1}{2}\left(r^{\alpha v+\beta}\left(S_{k}^{\sigma}\right)-\left(\alpha v\left(S_{k-1}^{\sigma}\right)+\sum_{i \in S_{k-1}^{\sigma}} \beta_{i}\right)\right. \\
& \left.-\left(\alpha v(\sigma(k))+\beta_{\sigma(k)}\right)\right) \\
= & \alpha\left(v(\{\sigma(k)\})+\frac{1}{2}\left(r\left(S_{k}^{\sigma}\right)-v\left(S_{k-1}^{\sigma}\right)-v(\{\sigma(k)\})\right)\right)+\beta_{\sigma(k)} \\
= & \alpha s_{\sigma(k)}^{\sigma}(v)+\beta_{\sigma(k)}
\end{aligned}
$$

For $k=1$, this is obvious.

From claim 1 and claim 2 it immediately follows that $\Psi_{i}(\alpha v+\beta)=\alpha \Psi_{i}(v)+\beta_{i}$ for all $i \in N$.

Theorem 1 Let $f: T U^{N} \longrightarrow \mathbb{R}^{N}$. Then, $f$ equals the consensus value if and only if it satisfies efficiency, symmetry, the neutral dummy property and additivity.

Proof We first show that the consensus value satisfies those four properties. Efficiency, symmetry, and additivity are straightforward to check.

Now we show that the consensus value satisfies the neutral dummy property. By relative invariance with respect to strategic equivalence (Lemma 1), it suffices to prove that the consensus value $\Psi$ satisfies the neutral dummy property for zero-normalized games. Let $v \in T U^{N}$ be zero-normalized and $i \in N$ a dummy in $v$. It suffices to show that $\Psi_{i}(v)=\frac{v(N)}{2|N|}$. This is readily checked for the case $|N|=2$.

We proceed by induction on the number of players using the recursive formula provided in Proposition 2. Suppose that for any zero-normalized game 
$v \in T U^{N^{\prime}}$ with $\left|N^{\prime}\right| \geq 2, \Psi_{i}(v)=\frac{v\left(N^{\prime}\right)}{2\left|N^{\prime}\right|}$ for a dummy player $i$ in $\left(N^{\prime}, v\right)$. Now consider a zero-normalized game $(N, v)$ with $|N|=\left|N^{\prime}\right|+1$. Let $i$ be a dummy player in $(N, v)$. Obviously, $v(\{i\})+\frac{v(N)-v(N \backslash\{i\})-v(\{i\})}{2}=0$.

Then, by Eq. (1), it suffices to show that

$$
\sum_{j \in N \backslash\{i\}}\left(\Psi_{i}\left(N \backslash\{j\},\left.v\right|_{N \backslash\{j\}}\right)+\frac{1}{|N|-1} \cdot \frac{v(N)-v(N \backslash\{j\})-v(\{j\})}{2}\right)=\frac{v(N)}{2} .
$$

Since player $i$ is also a dummy in the subgame $\left(N \backslash\{j\},\left.v\right|_{N \backslash\{j\}}\right)$, the induction hypothesis implies that $\Psi_{i}\left(N \backslash\{j\},\left.v\right|_{N \backslash\{j\}}\right)=\frac{v(N \backslash\{j\})}{2(|N|-1)}$. Then,

$$
\begin{aligned}
& \sum_{j \in N \backslash\{i\}}\left(\Psi_{i}\left(N \backslash\{j\},\left.v\right|_{N \backslash\{j\}}\right)+\frac{1}{|N|-1} \cdot \frac{v(N)-v(N \backslash\{j\})-v(\{j\})}{2}\right) \\
& =\frac{\sum_{j \in N \backslash\{i\}} v(N \backslash\{j\})}{2(|N|-1)}+\frac{(|N|-1) v(N)-\sum_{j \in N \backslash\{i\}} v(N \backslash\{j\})}{2(|N|-1)} \\
& =\frac{v(N)}{2}
\end{aligned}
$$

Conversely, let $f: T U^{N} \longrightarrow \mathbb{R}^{N}$ satisfy efficiency, symmetry, and the neutral dummy property. It easily follows that $f$ is uniquely determined for (multiples of) unanimity games. Hence requiring a solution $f$ to be additive too, it follows that $f$ is uniquely determined for any game in $T U^{N}$, since the class of unanimity games $\left\{\left(N, u_{T}\right) \mid T \in 2^{N} \backslash\{\emptyset\}\right\}$ constitutes a basis of $T U^{N}$.

We now provide an alternative characterization for the consensus value by means of the transfer property.

The transfer property (Dubey 1975) in some sense substitutes for additivity. It is defined as follows. For any two games $v, w \in T U^{N}$, we first define the games $(v \vee w)$ and $(v \wedge w)$ by $(v \vee w)(S)=\max \{v(S), w(S)\}$ and $(v \wedge w)(S)=$ $\min \{v(S), w(S)\}$ for all $S \subset N$. Let $f: T U^{N} \longrightarrow \mathbb{R}^{N}$ be a solution concept on the class of TU games. Then, $f$ satisfies the transfer property if $f(v \vee w)+f(v \wedge w)=$ $f(v)+f(w)$ for all $v, w \in T U^{N}$. Dubey (1975) characterized the Shapley value as the unique value on the class of monotonic simple games satisfying efficiency, symmetry, the dummy property, and transfer property. Feltkamp (1995) generalized this result to the class of all TU games. More specifically, the Shapley value is the unique value on the class of TU games satisfying efficiency, symmetry, the dummy property and the transfer property (cf. Feltkamp (1995), (p.134, Theorem 9.1.5)).

We now have an alternative characterization of the consensus value for TU games.

Theorem 2 The consensus value is the only one-point solution on the class of $T U$ games that satisfies efficiency, symmetry, the neutral dummy property and the transfer property. 
Proof As is well known, a solution concept $f: T U^{N} \longrightarrow \mathbb{R}^{N}$ satisfying additivity on $T U^{N}$ also satisfies the transfer property on $T U^{N}$. Therefore, the consensus value satisfies the transfer property. In addition, requiring a solution concept $f: T U^{N} \longrightarrow \mathbb{R}^{N}$ to satisfy efficiency, symmetry, and the neutral dummy property, it easily follows that $f$ is uniquely determined for (multiples of) unanimity games. Moreover, by Feltkamp (1995), (Lemma 9.1.4), it also follows that if the solution concept $f$ satisfies the transfer property too, it is uniquely determined for any game in $T U^{N}$.

Next we provide a third characterization for the consensus value, based on an idea similar to Myerson (1980)'s balanced contribution property for the Shapley value.

Let $f: T U^{N} \longrightarrow \mathbb{R}^{N}$ be a one-point solution concept. We say $f$ satisfies the equal welfare loss property ${ }^{6}$ if for any two players $i, j \in N$,

$$
\begin{aligned}
& f_{i}(N, v)-\left(\frac{1}{|N|-1} \frac{v(N)-v(N \backslash\{j\})-v(\{j\})}{2}+f_{i}\left(N \backslash\{j\},\left.v\right|_{N \backslash\{j\}}\right)\right) \\
& \quad=f_{j}(N, v)-\left(\frac{1}{|N|-1} \frac{v(N)-v(N \backslash\{i\})-v(\{i\})}{2}+f_{j}\left(N \backslash\{i\},\left.v\right|_{N \backslash\{i\}}\right)\right)
\end{aligned}
$$

for all $v \in T U^{N}$.

Below we explain the first part of the equation. The first term is simply how much player $i$ can get in game $(N, v)$ according to the solution concept $f$. Now we consider how much player $i$ should obtain in case player $j$ leaves the grand coalition $N$. As $j$ leaves, no doubt that player $i$ can get at least $f_{i}\left(N \backslash\{j\},\left.v\right|_{N \backslash\{j\}}\right)$ based on the remaining coalition $N \backslash\{j\}$. However, in the meantime, the leaving of player $j$ also raises the question how to deal with the surplus $v(N)-v(N \backslash\{j\})-v(\{j\})$ that has been jointly created by $j$ and $N \backslash\{j\}$. We argue that it should be equally shared between these two parties. So, $N \backslash\{j\}$ gets half of it and each player in $N \backslash\{j\}$ gets an equal share of this half. Hence the first part of the equation can be understood as the welfare loss of player $i$ due to the leaving of $j$. The equal welfare loss property requires that the leaving of any player induces the same level of welfare loss for everybody.

Theorem 3 The consensus value is the only one-point solution on $T U^{N}$ that satisfies efficiency and equal welfare loss property.

Proof One can readily check that the consensus value satisfies efficiency and the equal welfare loss property.

To show that the consensus value is the unique solution satisfying both properties, we assume there are two solution concepts, $f$ and $f^{\prime}$, both satisfying the two properties. We then prove $f$ and $f^{\prime}$ to be identical by induction on the number of players of the game.

6 The equal welfare loss property can be formulated by means of the stand-alone reduce game $v^{-i}$. That is, $f$ is said to satisfy the equal welfare loss property if for any two players $i, j \in N$, $f_{i}(N, v)-f_{i}\left(N \backslash\{j\}, v^{-j}\right)=f_{j}(N, v)-f_{j}\left(N \backslash\{i\}, v^{-i}\right)$ for all $v \in T U^{N}$. 
Apparently, by efficiency, this claim holds for 1-player games. We now assume that $f$ and $f^{\prime}$ are equal for games with $n-1$ players and show that this also holds for an arbitrary $n$-player game. Let $(N, v)$ be a game with $n$ players. By the induction hypothesis, we know $f_{i}\left(N \backslash\{j\},\left.v\right|_{N \backslash\{j\}}\right)=f_{i}^{\prime}\left(N \backslash\{j\},\left.v\right|_{N \backslash\{j\}}\right)$ for all $i, j \in N, i \neq j$. Based on the equal welfare loss property, we have

$$
\begin{aligned}
& f_{i}(N, v)-f_{j}(N, v) \\
& \quad=f_{i}\left(N \backslash\{j\},\left.v\right|_{N \backslash\{j\}}\right)-f_{j}\left(N \backslash\{i\},\left.v\right|_{M \backslash i\}}\right)+\frac{v(N \backslash\{i\})+v(\{i\})-v(N \backslash\{j\})-v(\{j\})}{2(|N|-1)} \\
& \quad=f_{i}^{\prime}\left(N \backslash\{j\},\left.v\right|_{N \backslash\{j\}}\right)-f_{j}^{\prime}\left(N \backslash\{i\},\left.v\right|_{N \backslash\{i\}}\right)+\frac{v(N \backslash\{i\})+v(\{i\})-v(N \backslash\{j\})-v(\{j\})}{2(|N|-1)} \\
& \quad=f_{i}^{\prime}(N, v)-f_{j}^{\prime}(N, v)
\end{aligned}
$$

for all $i, j \in N, i \neq j$. Therefore, $f_{i}(N, v)-f_{i}^{\prime}(N, v)=f_{j}(N, v)-f_{j}^{\prime}(N, v)$ for all $i, j \in N$. Now we fix $i$ and sum over $j \in N$ : due to efficiency, $\sum_{j \in N}\left(f_{j}(N, v)-f_{j}^{\prime}(N, v)\right)=$ 0 . Hence $f_{i}(N, v)=f_{i}^{\prime}(N, v)$ for all $i \in N$.

Yet, an alternative characterization of the consensus value by means of individual rationality and a type of monotonicity is provided in van den Brink et al. (2005).

\subsection{Generalizations}

Theorem 4 provides an explicit relationship between the consensus value and the Shapley value.

Theorem 4 For every $v$ in $T U^{N}$ it holds that

$$
\Psi(v)=\frac{1}{2} \Phi(v)+\frac{1}{2} E(v),
$$

where $\Phi(v)$ denotes the Shapley value of $v$ while $E(v)$ denotes the equal surplus solution of $v$, i.e., $E_{i}(v)=v(\{i\})+\frac{v(N)-\sum_{j \in N} v(\{j\})}{|N|}$ for all $i \in N$.

Proof It is readily shown that $f(v):=\frac{1}{2} \Phi(v)+\frac{1}{2} E(v)$ satisfies the four characterizing properties: efficiency, symmetry, neutral dummy property and additivity.

Note that Theorem 1 and Theorem 4 are equivalent. $^{7}$

The fact that the consensus value equals the average of the Shapley value and the equal surplus solution further reflects that the value takes a neutral stand between the two polar opinions of utilitarianism and egalitarianism, and

\footnotetext{
7 We would like to stress that the proof of Theorem 4 without the help of Theorem 1 will rely on lengthy combinatorial arguments.
} 
balances the tensions of the four fundamental principles of distributive justice as discussed in Sect. 3.1.

Based on Theorem 4, we can interpret the solution of the glove game in Example 2 by means of the four fundamental principles of distributive justice: The reward principle and the fitness principle have a tendency to assign more to the left-hand glove owner whereas the compensation and the exogenous rights principles favor equal allocation to all players. When they are in balance, we obtain the consensus value.

Dependent upon the tradeoff between the two polar forces within a society, the final bargaining result may lie in a position either closer to the Shapley value or the equal surplus solution. Thus, adopting a particular point between the two values can well reflect a society's propensity to the principles of distributive justice. Allowing for different propensities we obtain the following generalization of the consensus value.

Let $v \in T U^{N}$. We define the $\alpha$-remainder, with respect to an order $\sigma \in \Pi(N)$ and $\alpha \in[0,1]$, recursively by

$r^{\alpha}\left(S_{k}^{\sigma}\right)= \begin{cases}v(N) & \text { if } k=|N| \\ v\left(S_{k}^{\sigma}\right)+(1-\alpha)\left(r^{\alpha}\left(S_{k+1}^{\sigma}\right)-v\left(S_{k}^{\sigma}\right)-v(\{\sigma(k+1)\})\right) & \text { if } k \in\{1, \ldots,|N|-1\} .\end{cases}$

Correspondingly, the individual $\alpha$-remainder vector $\left(s^{\sigma}\right)^{\alpha}(v)$ is the vector in $\mathbb{R}^{N}$ defined by

$$
\left(s^{\sigma}\right)_{\sigma(k)}^{\alpha}(v)= \begin{cases}v(\{\sigma(k)\})+\alpha\left(r^{\alpha}\left(S_{k}^{\sigma}\right)-v\left(S_{k-1}^{\sigma}\right)-v(\{\sigma(k)\})\right) & \text { if } k \in\{2, \ldots,|N|\} \\ r^{\alpha}\left(S_{1}^{\sigma}\right) & \text { if } k=1\end{cases}
$$

Definition 2 For every $v \in T U^{N}$ and $\alpha \in[0,1]$, the $\alpha$-consensus value $\Psi^{\alpha}(v)$ is defined as the average over the set $\Pi(N)$ of all bijections, of the individual $\alpha$-remainder vectors, i.e.,

$$
\Psi^{\alpha}(v)=\frac{1}{|N| !} \sum_{\sigma \in \Pi(N)}\left(s^{\sigma}\right)^{\alpha}(v)
$$

Note that the consensus value corresponds to the case $\alpha=\frac{1}{2}$.

By extending the arguments on the neutral dummy property as mentioned in Sect. 3.1, one can define the $\alpha$-dummy property of a one-point solution concept $f: T U^{N} \longrightarrow \mathbb{R}^{N}$ by $f_{i}(v)=\alpha v(\{i\})+(1-\alpha)\left(v(\{i\})+\frac{v(N)-\sum_{j \in N} v(\{j\})}{|N|}\right)$ for all $v \in T U^{N}$ and every dummy player $i \in N$ with respect to $v$. Similarly, we say that $f$ satisfies the $\alpha$-equal welfare loss property if for any two players $i, j \in N$, 


$$
\begin{aligned}
& f_{i}(N, v)-\left(\frac{(1-\alpha)(v(N)-v(N \backslash\{j\})-v(\{j\}))}{|N|-1}+f_{i}\left(N \backslash\{j\},\left.v\right|_{N \backslash\{j\}}\right)\right) \\
& =f_{j}(N, v)-\left(\frac{(1-\alpha)(v(N)-v(N \backslash\{i\})-v(\{i\}))}{|N|-1}+f_{j}\left(N \backslash\{i\},\left.v\right|_{N \backslash\{i\}}\right)\right)
\end{aligned}
$$

for all $v \in T U^{N}$. Then, we obtain the following theorem.

Theorem 5 (a) The $\alpha$-consensus value $\Psi^{\alpha}$ is the unique one-point solution concept on $T U^{N}$ that satisfies efficiency, symmetry, the $\alpha$-dummy property and additivity.

(b) The $\alpha$-consensus value $\Psi^{\alpha}$ is the unique function that satisfies efficiency, symmetry, the $\alpha$-dummy property and the transfer property over the class of $T U$ games.

(c) For any $v \in T U^{N}$, it holds that

$$
\Psi^{\alpha}(v)=\alpha \Phi(v)+(1-\alpha) E(v) .
$$

(d) The $\alpha$-consensus value $\Psi^{\alpha}$ is the unique function that satisfies efficiency and the $\alpha$-equal welfare loss property over the class of TU games.

Proof Note that, analogous to Lemma 1, one can readily show that the $\alpha$ consensus value satisfies relative invariance with respect to strategic equivalence. Then, for any $\alpha \in[0,1]$, one can verify (a) and (b) along the lines of the proofs of Theorem 1 and Theorem 2. Next, (c) can be established due to the fact that $f(v):=\alpha \Phi(v)+(1-\alpha) E(v)$ satisfies efficiency, symmetry, the $\alpha$-dummy property and additivity. The proof of (d) is analogous to that of Theorem 3.

The expression of the $\alpha$-consensus value as provided in part (b) of Theorem 5 is in the same spirit as the so-called compound measures in the context of digraph competitions (cf. Borm et al. 2002).

Next we characterize the whole class of $\alpha$-consensus values by means of the bound dummy proportionality. A one-point solution concept $f: T U^{N} \longrightarrow \mathbb{R}^{N}$ satisfies the bound dummy proportionality if $0 \leq \frac{f_{i}(v)}{v(N)}=\frac{f_{j}(w)}{w(N)} \leq \frac{1}{|N|}$ for all zero-normalized games $v, w \in T U^{N}$, where $v(N) \neq 0$ and $w(N) \neq 0$, and every dummy player $i$ in game $v$ and every dummy player $j$ in game $w$, and $f_{i}(v)=0$ for all zero-normalized games $v \in T U^{N}$ with $v(N)=0$ and every dummy player $i$ in $v$.

Theorem 6 If a one-point solution concept on $T U^{N}$ satisfies efficiency, symmetry, the bound dummy proportionality, relative invariance with respect to strategic equivalence and additivity, then it belongs to the class of $\alpha$-consensus values.

Proof For any $\alpha \in[0,1]$, it is readily shown that the $\alpha$-consensus value satisfies the bound dummy proportionality due to part (c) of Theorem 5. Conversely, let a solution concept $f: T U^{N} \longrightarrow \mathbb{R}^{N}$ satisfy the bound dummy proportionality. Note that the bound dummy proportionality implies that there exists a unique $\alpha \in[0,1]$ such that for every zero-normalized TU game $w$ we have 
$f_{i}(w)=\alpha \cdot 0+(1-\alpha) \frac{w(N)}{|N|}$ for every dummy player $i$ in $w$. Now take any $v \in T U^{N}$ and let $\bar{v}$ be the corresponding zero-normalized game, defined by $\bar{v}(S)=v(S)-\sum_{i \in S} v(\{i\})$ for all $S \subset N$. Next, requiring $f$ to be relative invariant with respect to strategic equivalence, it follows that

$$
\begin{aligned}
f_{i}(v) & =f_{i}(\bar{v})+v(\{i\}) \\
& =(1-\alpha) \frac{\bar{v}(N)}{|N|}+v(\{i\}) \\
& =(1-\alpha) \frac{v(N)-\sum_{j \in N} v(\{j\})}{|N|}+(\alpha+(1-\alpha)) v(\{i\}) \\
& =(1-\alpha)\left(v(\{i\})+\frac{v(N)-\sum_{j \in N} v(\{j\})}{|N|}\right)+\alpha v(\{i\})
\end{aligned}
$$

for every dummy player $i$ in $v$. Note specifically that this $\alpha$ does not depend on $v$. This implies that $f$ satisfies the $\alpha$-dummy property. What remains is obvious due to part (a) of Theorem 5 .

\section{An implementation of the consensus value}

In this section, we will study the consensus value from a non-cooperative perspective, inspired by the bidding mechanism to implement the Shapley value as proposed by Pérez-Castrillo and Wettstein (2001). An alternative mechanism to implement the consensus value is discussed in Ju and Wettstein (2006).

The currently proposed mechanism to implement the consensus value has the key feature that it allows for a two-level bidding. The first level is designed for the set of all players, i.e., $N=\{1, \ldots, n\}$. On this level, if a proposal is rejected, there is probability $\frac{1}{2}$ that the game completely breaks down and every player gets her stand-alone payoff, while there is probability $\frac{1}{2}$ that the game can reach the second level which is for the remaining $|N|-1$ players as the proposer on the first level leaves. The possibility of breakdown on the first level bidding makes the current mechanism different from the one proposed by Pérez-Castrillo and Wettstein (2001). On the second level, i.e., for the remaining $|N|-1$ players, the mechanism is identical to the one in Pérez-Castrillo and Wettstein (2001).

We now formally describe the bidding mechanism. If there is only one player $\{i\}$, she simply receives $v(\{i\})$. When there are two or more players, the mechanism is defined recursively. Given the rules of the mechanism for at most $|N|-1$ players, the mechanism for $N=\{1, \ldots, n\}$ proceeds in three stages.

Stage 1: Each player $i \in N$ makes $|N|-1$ bids $b_{j}^{i} \in \mathbb{R}$ with $j \neq i$. Hence, at this stage, a strategy for player $i$ is a vector $\left(b_{j}^{i}\right)_{j \neq i}$.

For each $i \in N$, define the net bid to player $i$ by $B^{i}=\sum_{j \neq i} b_{j}^{i}-\sum_{j \neq i} b_{i}^{j}$. Let $i^{*}$ be the player with the highest net bid: an arbitrary tie-breaking rule can be used in case of a non-unique maximizer. Once the winner $i^{*}$ has been determined, player $i^{*}$ pays every player $j \in N \backslash\left\{i^{*}\right\}, b_{j}^{i^{*}}$. 
Stage 2: Player $i^{*}$ makes a vector of offers $x_{j}^{i^{*}} \in \mathbb{R}$ to every player $j \in N \backslash\left\{i^{*}\right\}$.

Stage 3: The players other than $i^{*}$, sequentially, either accept or reject the offer. If at least one player rejects it, then the offer is rejected. Otherwise, the offer is accepted.

If the offer is accepted, then each player $j \in N \backslash\left\{i^{*}\right\}$ receives $x_{j}^{i^{*}}$, and player $i^{*}$ receives $v(N)-\sum_{j \neq i^{*}} x_{j}^{i^{*}}$. Hence, the final payoff to player $j \neq i^{*}$ is $x_{j}^{i^{*}}+b_{j}^{i^{*}}$ while player $i^{*}$ receives $v(N)-\sum_{j \neq i^{*}} x_{j}^{i^{*}}-\sum_{j \neq i^{*}} b_{j}^{i^{*}}$.

If the offer is rejected, the game either breaks down with probability $\frac{1}{2}$, meaning that all players get their stand-alone values, or it continues, also with probability $\frac{1}{2}$. If the game continues, $i^{*}$ leaves the game and receives $v\left(\left\{i^{*}\right\}\right)$ whereas $N \backslash\left\{i^{*}\right\}$ proceeds to play a similar game with one player less bargaining over $v\left(N \backslash\left\{i^{*}\right\}\right)$. In case the game continues to the round for $N \backslash\left\{i^{*}\right\}$, the players follow similar rules as specified above for the case of $|N|$ players but the difference is that when an offer proposed by the player with the highest bid is rejected, the game will continue to the case of $|N|-2$ players with probability 1 . That is, if an offer made by the proposer, say $j^{*} \in N \backslash\left\{i^{*}\right\}$, is rejected, the game does not break down but $j^{*}$ leaves the game with $v\left(\left\{j^{*}\right\}\right)$ while the remaining players $N \backslash\left\{i^{*}, j^{*}\right\}$ continues to play the game. From now on, this rule applies to every possible round of bidding when an offer made by the corresponding proposer is rejected.

We will show that for any zero-monotonic game $v \in T U^{N}$ (i.e., $v(S) \geq$ $v(S \backslash\{i\})+v(\{i\})$ for all $S \subset N$ and all $i \in S)$, the subgame perfect equilibrium (SPE) outcomes of the bidding mechanism coincide with the payoff vector $\Psi(N, v)$ as prescribed by the consensus value. Note that the players in the mechanism are assumed to be risk neutral.

Theorem 7 The bidding mechanism implements the consensus value of a zeromonotonic game $(N, v)$ in SPE.

Proof Let $(N, v)$ be a zero-monotonic game. First we show that the consensus value is an SPE outcome. We explicitly construct an SPE that yields the consensus value as an SPE outcome. Consider the following strategies:

At stage 1, player $i \in N$ announces

$$
b_{j}^{i}=\frac{1}{2} \frac{v(N)-\sum_{k \in N} v(\{k\})}{|N|}+\frac{1}{2}\left(\Phi_{j}(N, v)-\Phi_{j}\left(N \backslash\{i\},\left.v\right|_{N \backslash\{i\}}\right)\right)
$$

for every $j \in N \backslash\{i\}$.

At stage 2, a proposer, player $i^{*}$, offers

$$
x_{j}^{i^{*}}=\frac{1}{2} v(\{j\})+\frac{1}{2} \Phi_{j}\left(N \backslash\left\{i^{*}\right\},\left.v\right|_{N \backslash\left\{i^{*}\right\}}\right)
$$

to every $j \in N \backslash\{i\}$. 
At stage 3, any player $j \in N \backslash\left\{i^{*}\right\}$ accepts any offer greater than or equal to $\frac{1}{2} v(\{j\})+\frac{1}{2} \Phi_{j}\left(N \backslash\left\{i^{*}\right\},\left.v\right|_{N \backslash\left\{i^{*}\right\}}\right)$ and rejects any offer strictly less than $\frac{1}{2} v(\{j\})+$ $\frac{1}{2} \Phi_{j}\left(N \backslash\left\{i^{*}\right\},\left.v\right|_{N \backslash\left\{i^{*}\right\}}\right)$.

Clearly, the combination of these strategies yields $\Psi(N, v)$ as the resulting payoff vector.

Note that all net bids equal zero by the balanced contribution property for the Shapley value (Myerson 1980).

Using zero-monotonicity and Theorem 1 in Pérez-Castrillo and Wettstein (2001) one readily verifies that the proposed strategy combination is indeed an SPE. Moreover, unicity of the SPE outcome can be established along similar lines as in the unicity proof of Pérez-Castrillo and Wettstein (2001).

Finally, we would like to note that one can modify and extend the above bidding mechanism for the consensus value in an appropriate way, i.e., the game either breaks down with probability $1-\alpha$ or continues with probability $\alpha$ in case of an offer being rejected at stage 3 of the first level bidding, to implement the $\alpha$-consensus value as discussed in Sect. 3.2.

Acknowledgements The authors thank René van den Brink, Eric van Damme, Dolf Talman and David Wettstein for helpful discussions and comments. We also appreciate the comments from the audience at the 2nd World Congress of the Game Theory Society in Marseille and the 7th International Meeting of the Society for Social Choice and Welfare in Osaka. In particular, we thank two anonymous referees and an associate editor for their valuable comments and constructive suggestions.

\section{References}

Borm P, van den Brink R, Slikker M (2002) An iterative procedure for evaluating digraph competitions. Ann Oper Res 109:61-75

van den Brink R, Funaki Y (2004) Axiomatizations of a class of equal surplus sharing solutions for cooperative games with transferable utility. Tinbergen Institute Discussion Paper, TI 2004136/1, Tinbergen Institute, Amsterdam/Rotterdam, The Netherlands

van den Brink R, Funaki Y, Ju Y (2005) Axiomatizations of the convex combinations of a class of equal surplus solutions and the shapley value, Manuscript, Free University Amsterdam, the Netherlands

Driessen TSH, Funaki Y (1991) coincidence of and collinearity between game theoretic solutions. OR Spektrum 13:15-30

Dubey P (1975) On the uniqueness of the shapley value. Int J Game Theory 4:229-247

Feltkamp V (1995) Cooperation in controlled network structures. Ph.D. Dissertation, Tilburg University

Ju Y (2004) The consensus value for games in partition function form. CentER Discussion Paper, 2004-60, Tilburg University, The Netherlands. To appear in International Game Theory Review

Ju Y, Ruys P, Borm P (2004) Compensating losses and sharing surpluses in project-allocation situations. CentER Discussion Paper, 2004-06, Tilburg University

Ju Y, Borm P (2005) Externalities and compensation: primeval games and solutions. Keele Economics Research Paper, 2005-05, Keele University, UK

Ju Y, Wettstein D (2006) Implementing cooperative solution concepts: a generalized bidding approach. Keele Economics Research Paper, 2006-06, Keele University

Maschler M, Owen G (1989) The consistent shapley value for hyperplane games. Int J Game Theory 18:389-407

Moulin H (2003) Fair division and collective welfare. MIT Press, Cambridge, MA 
Myerson R (1980) Conference structures and fair allocation rules. Int J Game Theory 9:169-182

Pérez-Castrillo D, Wettstein D (2001) Bidding for the surplus: a non cooperative interpretation of the shapley value. J Econ Theory 100:274-294

Quant M, Borm P, Maaten R (2005) A concede-and-divide rule for Bankruptcy problems. CentER Discussion Paper, 2005-20, Tilburg University

Rubinstein A (1982) Perfect equilibrium in a bargaining model. Econometrica 50:97-109

Shapley LS (1953) A value for $n$-person games. In: Kuhn H, Tucker AW (eds) Contributions to the theory of games, vol. II. Princeton University Press, Princeton, NJ 